\title{
Ofidios terrenales y sobrenaturales, símbolos de poder de los ajawtaak de Kaan
}

\section{( Pablo A. Mumary Farto*}

\section{Resumen}

Desde momentos tempranos, los señores de Kaan vincularon a su grupo de poder con un animal de fuerte calado simbólico en la colectividad prehispánica: la serpiente. Los portadores del glifo emblema de la Cabeza de Serpiente se convirtieron en una dinastía dominante durante buena parte del Clásico y su signo de identidad aparece recurrentemente en las narrativas jeroglíficas conservadas en los monumentos de piedra. Asimismo, tres ofidios más fueron empleados por los ajawtaak de Kaan como parte de una estrategia de refuerzo de la autoridad dinástica: la primera procedente de una tradición simbólica anterior y relacionada con la guerra, Waxaklajunubaah Chan, la serpiente teotihuacana; la segunda, conocida como Serpiente acuática ligada a la élite gobernante y quizá asociada al control de los recursos acuíferos; y la tercera, una entidad anímica muy poderosa vinculada a los gobernantes dinásticos tardíos pero procedente de una tradición anterior, Xukub Chij Chan. En este trabajo, a través de un estudio iconográfico y epigráfico de monumentos de piedra y objetos cerámicos, analizaré los ofidios a los que se vincularon los gobernantes de la dinastía Kaan y cuáles fueron las posibles razones que les llevaron a ello.

\section{Earthly and supernatural snakes, symbols of power of the Kaan ajawtaak}

\begin{abstract}
From early times, Kaan lords linked their power group to a strongly symbolic prehispanic animal: the snake. Those who wore the Snake's Head emblem glyph became a dominant dynasty during the Classic Period and this identity sign recurrently appears in hieroglyphic narratives preserved in rock monuments. Likewise, the Kaan ajawtaak used other three ophidiids to reinforce dynastic authority: the first one derives from a previous symbolic tradition related to war, the Teotihuacan serpent; the second one, known as the aquatic serpent, associated with the ruling elite and probably also with aquatic resource control; and the third one, a very powerful animistic entity related with late dynastic rulers but stemming from a previous tradition, Xukub Chij Chan. Using an epigraphic and iconographic study of stone monuments and pottery, I will analyze the reasons regarding the relationship between these ophidiids and Kaan dynasty rulers.
\end{abstract}

* Instituto de Investigaciones Antropológicas, Universidad Nacional Autónoma de México (UNAM). Av. Universidad 1900, 38, 2, Barrio Oxtopulco-Universidad (CP 04310), Coyoacán, Ciudad de México, México. E-mail: pablomumaryfarto@hotmail.com
Recibido:

13 de marzo de 2018

Aceptado:

14 de septiembre de 2018

Palabras clave

Serpiente

Kaan

Wahy

Glifo emblema

Simbolismo

Iconografía

Keywords

Snake

Kaan

Wahy

Emblem glyph

Symbolism

Iconography 
1. En esta ocasión, a lo largo de todo el escrito utilizaré el sufijo plural -taak en lugar de la forma enclítica $-o$ 'ob, siendo consciente que, por una parte, el empleo del sufijo plural en las diferentes lenguas mayas es algo opcional (Kettunen y

Helmke, 2014, p. 135) y, por otra, que es probable que el uso de-taak se refiera a un colectivo más que a una forma plural en sí (Law y Stuart, 2017, p. 145).

2. La transliteración, la transcripción y la traducción son las tres fases fundamentales del proceso analítico epigráfico. En la primera se utilizan los guiones para separar los diferentes elementos que componen el cartucho jeroglífico y se disponen en negritas tal y como están escritos los signos diferenciando los logogramas en mayúsculas y las sílabas en minúsculas, en la transcripción, se representa en cursiva (con reconstrucciones indicadas entre corchetes) tal y como deberían ser leídos los elementos representados. Por último, la traducción propuesta se reproduce entre comillas simples.

\section{La cabeza de serpiente, el glifo emblema}

Durante los siglos VII y VIII d.C., la realidad político-social de las Tierras Bajas mayas se vio marcada por una creciente ola de cruentos enfrentamientos entre diferentes entidades políticas encabezadas, en muchos casos, por dinastías gobernantes principales o grupos de poder subordinados a las anteriores. En este contexto, se produjo el surgimiento de alianzas que favorecían el control de regiones geoestratégicas por parte de los k'uhulajawtaak ${ }^{1}$ asociados a diferentes glifos emblemas repartidos por todo el territorio.

Identificados en 1958 (Berlin, 1958) los glifos emblema funcionaron, probablemente, como identificadores de dinastías concretas, aunque en ocasiones pueden tener también el valor de determinantes topográficos (Biró, 2011, p. 291) o incluso referirse a localizaciones míticas (Helmke, 2012).

Barthel (1968) fue el primer investigador que propuso una asociación entre el jeroglífico que Thompson había clasificado como el signo T764 (Figura 1) en su catálogo de 1962, A Catalog of Maya Hieroglyphs, (Thompson, 1962) con el día chikchan del Tzolk'iny con el signo chan, "serpiente". Dada la distribución del T764 en los monumentos de piedra conocidos hasta ese momento, Barthel consideró que este glifo emblema debía estar relacionado con alguna entidad política muy importante. Esto permitió que pocos años después Joyce Marcus (Marcus, 1973) partiendo del estudio de la Estela 10 de Ceibal y la Estela A de Copán, propusiera que el signo T764 era en realidad el glifo emblema de Calakmul, entidad política situada en el sureste del actual estado mexicano de Campeche.

El estudio etimológico realizado por Kaufman y Justeson en su obra del $2003 A$ Preliminary Mayan Etymological Dictionary, mostró que el vocablo chan es la palabra con la se refieren en muchas lenguas mayas a 'culebra o serpiente', presentando variantes como kan (en Itzá), kaan (en Yucateco) o incluso chon (en Tzotzil), aunque todas procederían de una raíz común Protomaya: kaan. Por tanto, el signo principal del compuesto jeroglífico que había sido propuesto como glifo emblema de Calakmul y que se representaba con una cabeza de serpiente, debía ser leído chan, sin embargo dicho signo se acompaña de un prefijo $\boldsymbol{k a}$ - que modifica la lectura chan (de filiación lingüística cholana) por kaan, la cual además de mostrar una fonología yucatecana le confiere un valor arcaico al glifo emblema en cuestión (Lacadena y Wichmann, 2002). Asimismo, la presencia en muchos ejemplos de un complemento fonético con vocal que puede variar, llevó a distintos investigadores a proponer una posible lectura del mismo como - $\boldsymbol{u}^{\prime} \boldsymbol{l}$ (que aparece también en otros glifos emblema) (Beliaev, 2015) lo que daría lugar a la forma kanu'l entendida como 'abundancia de serpiente' por la presencia del complemento. También, recientemente Helmke y Kupprat han propuesto que en algunas circunstancias kanu'l podría estar haciendo referencia a "una cueva con ciertos elementos acuáticos que estaba estrechamente relacionada con el mito del dios del maíz" (Helmke y Kupprat, 2017, p. 120).

Con base en lo anterior, a lo largo del presente escrito, se utilizará la forma arcaica Kaan para designar a la dinastía al no existir, de momento, un consenso generalizado entre los especialistas en cuanto al término kanu'l. Por tanto, el glifo emblema (Figura 2) compuesto fundamentalmente por cuatro signos diferenciados (más el complemento fonético con vocal variable señalado anteriormente) se podría transliterar, transcribir y traducir de la siguiente manera: $K^{\prime} \boldsymbol{U H}-\boldsymbol{k a}-\boldsymbol{K A N}-\boldsymbol{A J A W}, k^{\prime} u h[u l]$ Kaanajaw, 'sagrado gobernante de Kaan'2.

En otros estudios se han identificado iconográficamente distintos tipos de serpientes consideradas sagradas entre los mayas (De la Garza, 2001), siendo estas además elementos 


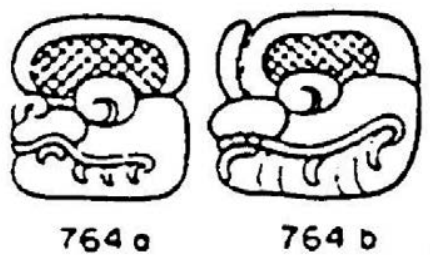

Figura 1. Signo jeroglífico T764 (según Thompson, 1962, p. 455).

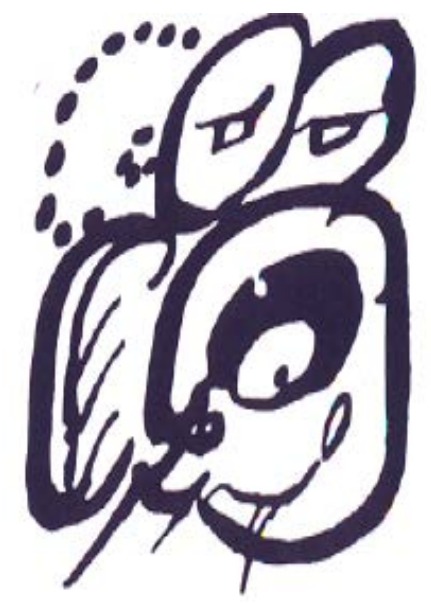

Figura 2. Glifo emblema de Kaan, cartucho jeroglífico $\mathrm{H}_{3}$ del vaso dinástico K6751 (según Martin, 1997, p. 2).

iconográficos recurrentes vinculados con el poder de los gobernantes representados a través de la toma o carga de la llamada barra ceremonial (un elemento iconográfico que se encuentra desde épocas tempranas y se perpetúa durante el Clásico Tardío) (Clancy, 1994) en la que se figuraba una sierpe bicéfala o no, con sus fauces abiertas en uno o en los dos extremos del artefacto. En este sentido, algunos autores han reparado que a través del signo jeroglífico no podría determinarse la especie de serpiente representada (Zender y Stone, 2011) o incluso que el carácter principal no evoca a un ofidio en sí, sino que más bien tal y como indicó D’arcy Harrison "se trata de una composición fantástica de diferentes rasgos de criaturas distintas" (D’arcy Harrison, 2008, p. 119). Sin embargo, coincidiendo con la interpretación de Kettunen y Davis II, considero que probablemente el signo central represente una cabeza de Boa constrictor, especie de la familia Boidae, la cual se encuentra ampliamente distribuida por las Tierras Bajas mayas y que se caracteriza (Kettunen y Davis II, 2004), entre otros elementos, por su mandíbula compuesta por pequeños colmillos ganchudos, un detalle diagnóstico del carácter central del glifo emblema. De esta manera, la dinastía Kaan habría tomado como signo de identidad a un animal con una gran carga simbólica en el imaginario colectivo del periodo Clásico, la serpiente, misma que en palabras de Mercedes de la Garza representa:

(...) En la religión maya, la serpiente tiene una significación plurivalente: es celeste, terrestre e infraterrestre. Así, en tanto que otros animales simbolizan aspectos concretos de la naturaleza, la serpiente parece encarnar los grandes contrarios cósmicos y su armonía, que hace posible la existencia; parece simbolizar una energía sagrada de la que participan dioses y hombres; de ahí su omnipresencia en el arte plástico y los códices y el papel que desempeña en los mitos y en los ritos. (De la Garza, 2001, p. 146). 
Pero los trabajos que se desarrollaron en el sitio arqueológico de Calakmul, situado en el actual estado mexicano de Campeche, a partir de los proyectos de investigación de los años ochenta del siglo XX encabezados por William Folan y la Universidad Autónoma de Campeche (hasta comienzos de los noventa) y el Instituto Nacional de Antropología e Historia de México bajo la dirección del arqueólogo Ramón Carrasco Vargas (desde 1993 hasta el 2016), develaron que el glifo emblema de la Cabeza de Serpiente no se registraba en los monumentos de la ciudad campechana hasta el siglo VII d.C. Empero, las labores arqueológicas desarrolladas en sitios del estado mexicano de Quintana Roo como Dzibanché (Nalda, 2004), Pol Box (Esparza Olguín y Pérez Gutiérrez, 2009) o El Resbalón (Esparza, 2012) expusieron registros jeroglíficos fechados para el Clásico Temprano en los que sí se podía identificar el glifo emblema con el signo T764. Esto aunado al aumento exponencial de inscripciones con la cabeza de serpiente que se descubrieron desde finales del siglo XX en muchas ciudades de las Tierras Bajas mayas, permitió deducir que, en el caso de este glifo emblema, funcionaba como un identificador de un grupo de poder destacado que tuvo una gran presencia e importancia en el contexto histórico del periodo Clásico, la dinastía Kaan, portadores del glifo emblema de la Cabeza de Serpiente. Asimismo, recientes descubrimientos que han tenido lugar en el sitio de Xunantunich (Belice) han permitido a Helmke y Awe (2016) develar un conflicto en el seno dinástico durante las primeras décadas del siglo VII d.C., cuya resolución fijó el establecimiento de Calakmul como nueva sede dinástica.

Además de en los monumentos de piedra, el glifo emblema de Kaan ha sido identificado en diversos objetos cerámicos de estilo códice, dicha categoría pertenece al grupo cerámico Zacatal o Palmar pudiendo ubicar su elaboración durante la fase cerámica Tepeu II entre los años 672-751 d.C. (Reents-Budet, Boucher, Palomo, Bishop y Blackman, 2011). Como veremos a continuación, el estudio de estos materiales es fundamental para poder analizar la vinculación del grupo dinástico con el animal presentado, la serpiente, símbolo de poder no sólo en el plano de existencia terrenal sino también más allá de este, personificada también a través de los wahy (entidades anímicas vinculadas a los gobernantes más prominentes del periodo Clásico) y otros ofidios sobrenaturales.

\section{Waxaklajunubaah Chan y la Serpiente acuática}

Como mostré previamente, la dinastía Kaan tomó como elemento iconográfico representativo y central en su glifo emblema una cabeza de serpiente, imitando posiblemente la apariencia de una Boa constrictor. Pero además, los k’uhulajawtaak de Kaan se vincularán simbólicamente con tres ofidios de características sobrenaturales a través de los cuales se revestirán de poder y ensalzarán la dinastía en contextos históricos determinados, Waxaklajunubaah Chan, la Serpiente acuática y el wahy Xukub Chij Chan.

\section{a) Waxaklajunubaah Chan}

El conocido como Waxaklajunubaah Chan (Figura 3) es un ente serpentino de carácter bélico de origen teotihuacano (Taube, 1992) pues de allí proceden las referencias más tempranas conocidas del ser sobrenatural que se caracteriza por su largo hocico, la cresta que lo decora y los dientes curvos, elementos que quizá, tal y como expuso Taube "estén representando a una serpiente de cascabel ya que en ocasiones se identifica un colmillo culminado por una figura circular, posiblemente el veneno" (Taube, 1992, p. 13) del que hace gala el poderoso y temido ofidio. Este motivo iconográfico fue asimilado y utilizado en muchas imágenes de las Tierras Bajas mayas, destacando su representación en tocados que portan los principales gobernantes de las entidades políticas mayas del Clásico (como en la Estela 7 de Piedras Negras, entre otros ejemplos), que de esta manera se personifican como los más importantes guerreros de su tiempo. En el caso de la dinastía Kaan son tres los gobernantes que se "apropiarán" de las cualidades de este 


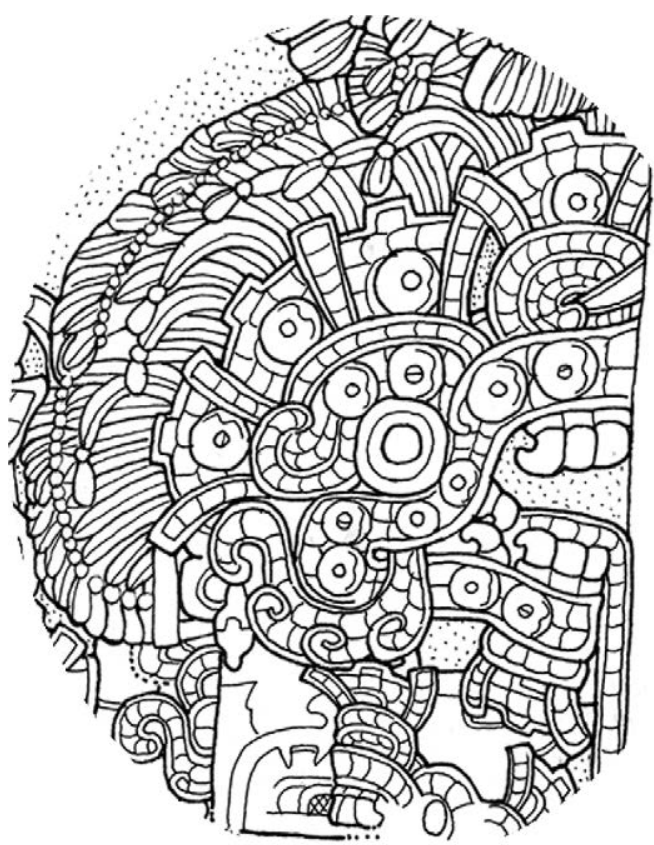

Figura 3. Representación de Waxaklajunub'aah Chan en el Dintel 2 del Templo I de Tikal (según Schele en Freidel, David, Schele y Parker, 1993, Figura 7.18).

ofidio de características bélicas y se harán representar personificando a dicha entidad, portando algunos de sus atributos en monumentos de piedra y objetos cerámicos: Yuhkno'm Chèn II, Yuhkno'm Yihchiaak K'ahk'y Yuhkno'm Took' K'awiil.

Yuhkno'm Yihchiaak K'ahk' tomó el poder de la dinastía en el año 686 d.C., heredando una situación político-social supuestamente favorable tras el gobierno de su antecesor Yuhkno'm Ch'̉n II. En el Bloque V de la Escalera Jeroglífica 2 de La Corona (sitio ubicado en Guatemala), la narrativa jeroglífica en los cartuchos D5-D6 indica que el k'uhulajaw de Kaan llega, posiblemente, a esta ciudad encarnando al ente Waxaklajunubaah Chan presentándose ante la colectividad ataviado con atributos reconocibles de la entidad. En el mismo sitio arqueológico se encontró la Estela 1 en la cual también se hace referencia en el cartucho $\mathrm{C} 4$ al gobernante de Kaan en un evento de celebración de fin de periodo 9.13.0.0.0 ( 16 de marzo del 692 d.C.), personificando a la serpiente de la guerra. Como indicó Stuart (2012), es probable que el gobernante se ataviase con rasgos distintivos de esta entidad bélica durante sus encuentros con los ajawtaak subordinados de su área de influencia regional, buscando reforzar su imagen y revalorizar a la dinastía al portar símbolos de poder procedentes de una tradición anterior, la teotihuacana, de este modo, el gobernante sería la representación terrenal de la poderosa entidad sobrenatural.

Su sucesor, Yuhkno'm Took Kawiil, fue nombrado como nuevo gobernante en algún momento alrededor del año 702 d.C., pues es la fecha más temprana que aparece en una estela erigida por él en Calakmul, la Estela 24. Con este gobernante se levantarán un gran número de monumentos de piedra en la ciudad y se llevará a cabo un programa de renovación de algunas de las estructuras más destacadas. Además, como mencioné anteriormente, es posible que durante su periodo de mandato fuese cuando se manufacturaron un gran número de objetos cerámicos estilo códice. Yuhkno'm Took' K'awiil se hizo representar en muchas de sus estelas como el gran guerrero y gobernante de su época y por eso, además de ser poseedor de títulos de importancia como kajlo’mte’3, se engalanará con los símbolos de poder de mayor importancia y los asociará a la dinastía desde momentos tempranos. En este sentido, cabe destacar la Estela 52 (Figura 4) de
3. A lo largo del escrito utilizaré la propuesta de Guillermo Bernal Romero para el término $\boldsymbol{K} \boldsymbol{A L}$ TE' o ka-lo-ma-TE' en la que se reconstruye la consonante aspirada velar /j/, dando lugar a la transcripción ka[j]lo'mte' con el significado de "quebrador de lanzas" (Bernal Romero, 2015, p. 22). 


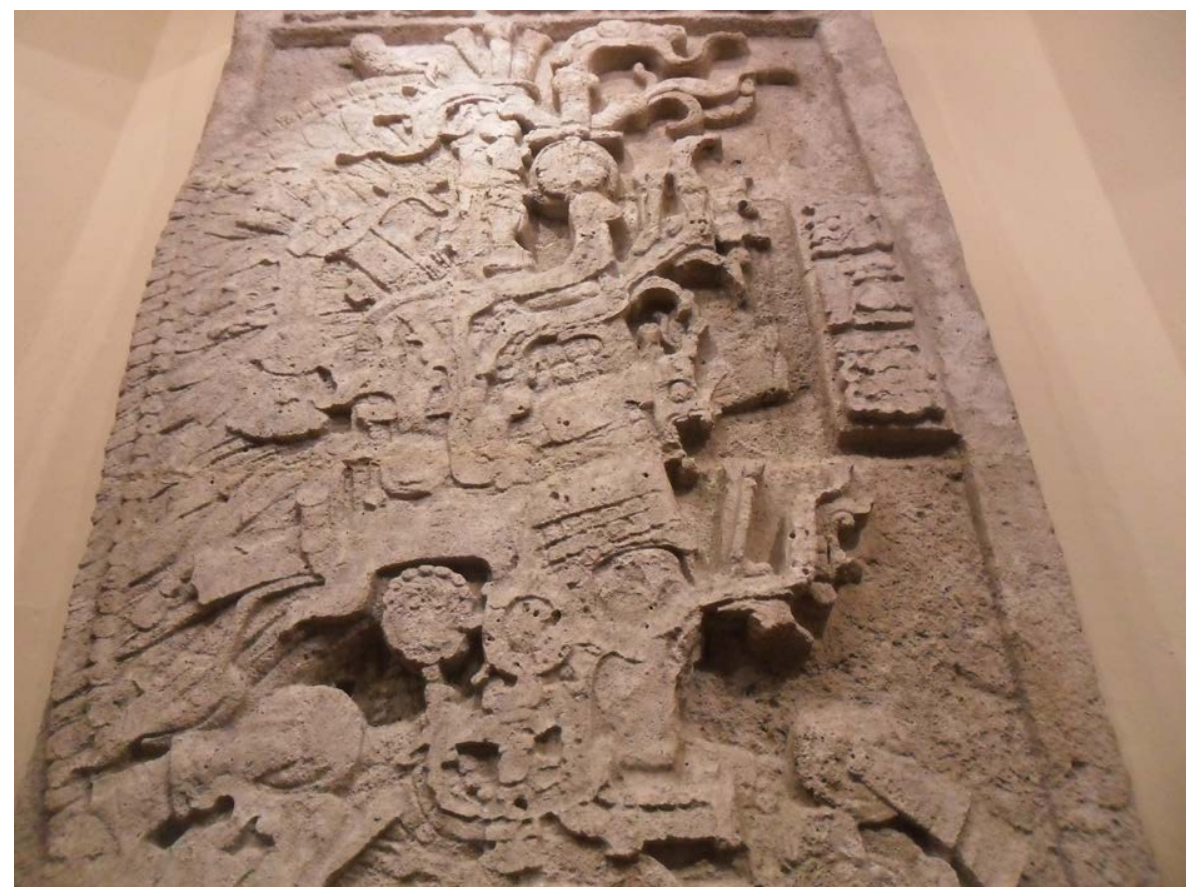

Figura 4. Estela 52 de Calakmul, Museo Dolores Olmedo, Ciudad de México. Foto del autor.

Calakmul en la que el ajaw, bajo su ostentoso tocado, viste un yelmo de teselas, un $k o ’ h a w$, de reminiscencia teotihuacanas relacionado además con la serpiente de la guerra, simbolizando su importancia como caudillo militar.

Las referencias más tempranas conocidas hasta la fecha en las que aparece el título de kajlo'mte' se han identificado en objetos vinculados al evento histórico conocido como "la entrada" (Stuart, 2000) durante el cual, grupos humanos procedentes de Teotihuacán se introducen en el siglo IV d.C., por las Tierras Bajas mayas quedando registro iconográfico y jeroglífico que atestigua dicha incursión (Estrada-Belli et al., 2009), un ejemplo destacado de ello se encuentra en la Estela 31 de Tikal. Asimismo, referencias al ko'haw como atuendo bélico se encuentran en representaciones de Teotihuacán y, posteriormente, en muchos monumentos del área maya ligado a los más importantes gobernantes y guerreros, como por ejemplo en el Panel 2 de Piedras Negras o la Estela 26 del mismo sitio.

Cabe señalar que en la llamada vasija Schaffhausen (Figura 5) en la que se encuentra representado el gobernante Yuhkno'm Chèn II, anterior a los dos presentados, pero cuya manufactura puede responder, tal y como indicó Prager (2004), a un encargo directo de Yihchiaak K'ahk o a los momentos finales de su gobierno, la tapadera remata con una figura de rasgos antropomorfos que porta, además de unas grandes orejeras, un curioso tocado zoomorfo. La iconografía y las grandes fauces abiertas permiten reconocer la imagen de Waxaklajunubaah Chan, la serpiente de guerra teotihuacana.

\section{b) La serpiente acuática}

Conocida en la literatura académica también por otros nombres como: Water lily serpent, Water lily monster o Water serpent (ésta última denominación se debe al investigador Stuart, 2007), su antropónimo durante el Clásico podría haber sido: Yax Chit Jun Witz' Nah Kan (Kettunen y Helmke, 2013, p. 23). Este ser serpentino es una de las maneras de representar, de forma personificada, al agua destacando entre sus 


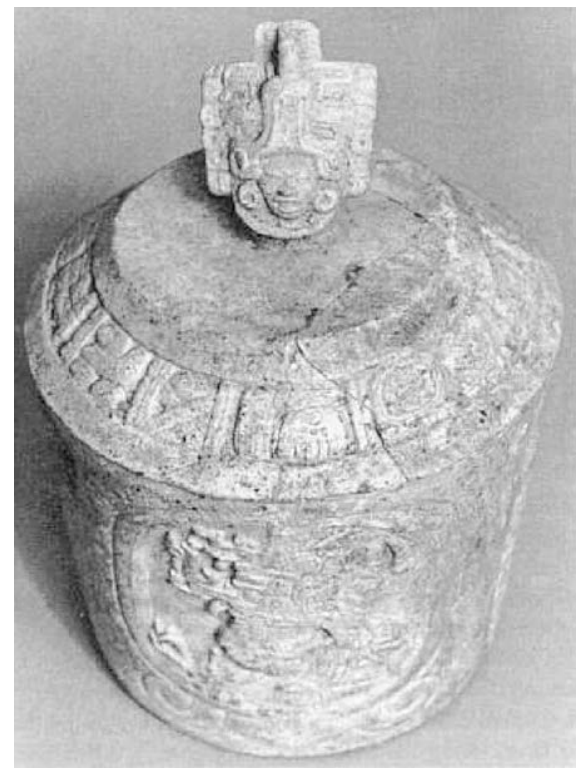

Figura 5. Vasija Schaffhausen (según Prager, 2004, Figura 5).

atributos una larga mandíbula superior que suele acabar en forma de vírgula con colmillos pronunciados y la presencia de un nenúfar del cual, en algunos ejemplos, pende un pescado (Figura 6). En su versión jeroglífica se pueden encontrar variantes con un signo principal que representa una cabeza serpentina completado con un elemento imix, $h a$ ' o haab, relacionados con el agua. Su vinculación con dicho líquido se refuerza al ser figurada, en muchas ocasiones, con bandas acuáticas y acompañarse del jeroglífico polaw, 'manantial, océano, mar' (Zender y Stone, 2011, p. 141), sobre todo en vasijas cerámicas. Además, la presencia del logograma WITZ', identificado por Stuart como "cascada", tal y como se atestigua en lengua Chorti', permite la asociación del ente con ciertos movimientos de agua.

Cabe señalar que esta entidad no es exclusiva de los Kaan pues conocemos su presencia en monumentos de otras ciudades de las Tierras Bajas acompañando a las élites tanto en las representaciones como en las narrativas jeroglíficas, como por ejemplo en Yaxchilán en el Dintel 1 donde aparece nombrada la entidad, en Bonampak en la Estela 2 también se muestra en el tocado de una de las figuras representadas o en Machaquilá donde contamos con muchos ejemplos como la Estela 3 (Figura 7), entre otros casos. En ellos se puede intuir como sus gobernantes en determinadas ocasiones llegaban a "fusionarse" con el ente serpentino al vestir el ajaw un elaborado tocado con la conocida como "máscara de rayos X" (Velásquez García, 2007) con la faz del ofidio. De esta manera, la importancia que las dinastías del Clásico parecen haberle concedido a la Serpiente acuática queda también atestiguada para la dinastía Kaan, como veremos a continuación, en los diferentes monumentos en los que los gobernantes y otras entidades corporativas asociados a los k'uhulajawtaak se hacen representar acompañados del ente.

De los monumentos de piedra que se han conservado del gobernante Yuhkno'm Took' K'awiil destaca la presencia de la Serpiente acuática en cuatro de ellos, vinculada de una forma directa tanto con el ajaw como con la dinastía. Por un lado, en las estelas 51, 52 y 54 de Calakmul en las que Yuhkno’m Took' K'awiil personifica el poderío político-militar de los Kaan, sobresale la presencia de dicho ser en el elaborado tocado en la Estela 54, erigida por el gobernante en la misma fecha que la anterior (9.15.0.0.0, 20 de agosto del 731 d.C.), se representa, posiblemente, a una de las esposas del ajaw. Esta señora porta 


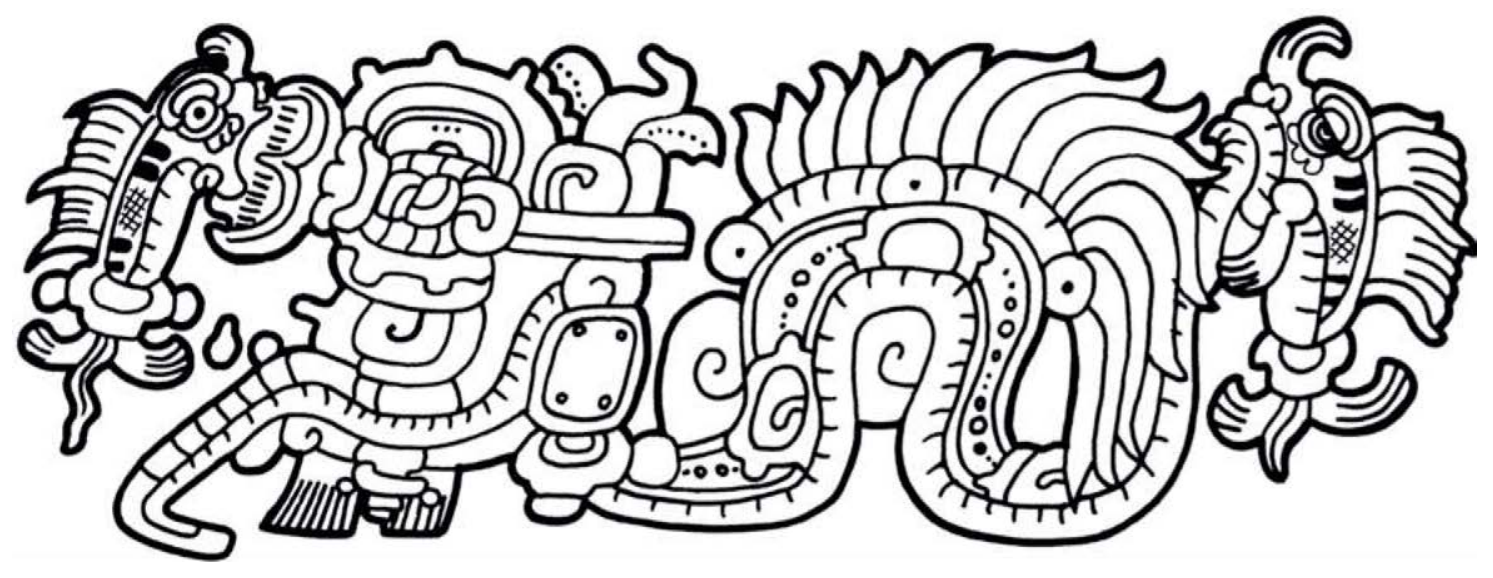

Figura 6. Serpiente acuática (según Zender y Stone, 2011, p. 140).

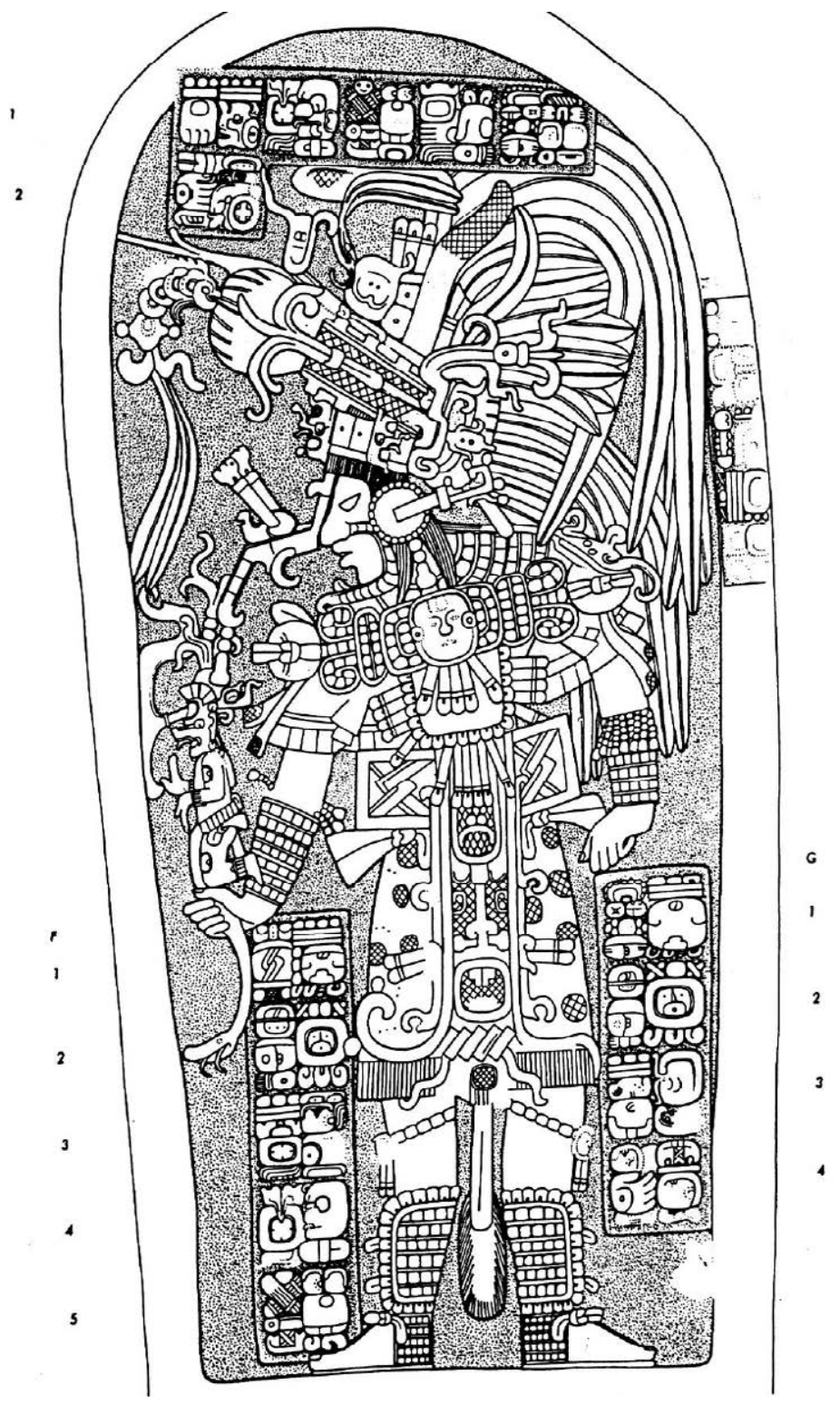

Figura 7. Estela 3 de Machaquilá (según Graham, 1967, p. 49). 
un elaborado tocado en el que, pese al desgaste del monumento, todavía se pueden apreciar el nenúfar y el pescado que suelen mostrarse en las representaciones de la Serpiente acuática; además, según Baron (2013, p. 387), en el monumento "aparece el nombre jeroglífico de la entidad formando parte de una larga lista de deidades patronas dinásticas durante un evento de tipo político-ritual”.

Por otro, en el Panel 6 de La Corona (Figura 8) —un monumento en el que se está fortaleciendo la alianza favorable y duradera entre los Kaan y los gobernantes de La Corona a través del relato de la llegada de mujeres de la élite del glifo emblema de la Cabeza de Serpiente (desde tiempos tempranos) con el fin de establecer uniones matrimoniales (Martin, 2008) - llama la atención en la parte izquierda de la composición, la representación en su forma completa de la Serpiente acuática, quizá como ente protectora de la mujer o como símbolo de poder asociado ya a la dinastía, sobre una estructura tipo templo que protege a una de estas señoras de Kaan enviadas a La Corona.

No debo dejar de señalar que es probable que las importantes dinastías del Clásico fortaleciesen su posición a través del control de un bien fundamental: el agua. Por ello, es significativo el hecho de que en el caso de los Kaan, tanto en Dzibanché (Quintana Roo, México) como Calakmul (Campeche, México) - las dos ciudades que fueron capitales dinásticas durante el Clásico-, se gozaba de este recurso natural a través de las grandes aguadas y acumulaciones de agua de las que se disponían (Domínguez y Folan, 1996). En este sentido, en la entidad política de Yookop en Quintana Roo, también conocida como Okop o La Aguada debido a una zona de acumulación de agua que se ha encontrado en el sitio arqueológico, se reportó una serie de bloques de piedra con inscripciones jeroglíficas (Shaw, Johstone y Krochock, 2001) que la situaban bajo la influencia de los ajawtaak de Kaan durante el gobierno de Testigo del Cielo (siglo VI d.C.). Aunque la información no queda clara debido a la falta de monumentos (Wren y Nygard, 2005, p. 172), los Bloques B, C y D (Figura 9) podrían mostrar la relación entre dicho gobernante y una mujer kajlo'mte' de nombre IxChakCh'én, quien entre sus títulos porta el logograma de la Serpiente acuática con un signo $T U N$ y un posible $N A L$, quizá haciendo referencia a un título toponímico asociado con las aguas.

Además, cabe advertir que este ente está relacionado con una de las deidades más importantes del panteón maya desde tiempos Preclásicos: el dios del Maíz. Ya en los murales de San Bartolo, sitio Preclásico situado en Guatemala, se observa una escena (en el mural del Edificio Sub1-A) en la que se encuentran Chaahk (deidad clásica de la lluvia y el rayo), el dios del Maíz y, posiblemente, la Serpiente acuática (Figura 10) en un lugar identificado como una cueva con todo el simbolismo que ello conlleva, al ser "la montañacueva el lugar de origen y morada de los ancestros", como indicó Carrasco Vargas (2012, p. 80), lo que denota la importancia de este ser en el panteón maya desde momentos tempranos. Finalmente, debo señalar que también el gobernante Yuhkno’m Chẻn II se vinculó con este ofidio de las aguas: en la Tumba 4 de la Subestructura II-B de Calakmul, que albergó el cuerpo de Yuhkno’m Yihchiaak K'ahk', se depositó una máscara de jadeíta, obsidiana y concha que contiene una inscripción en la parte inferior de su mandíbula derecha. Por el registro escriturario que se conserva en ella se puede intuir que el objeto debió pertenecer a Yuhkno'm Chẻn II, y que éste era a su vez la encarnación terrenal de la Serpiente acuática cuyo nombre aparece, de manera contraída (Figura 11), de la siguiente manera: $\boldsymbol{u}$-BAH YAX-CHIT-WITZ' yu-[ku]-no?-CH'EN-na, u ba[a]h Yax Chit Witz' Yu[h] kno['m] Chèn, 'Yuhkno'm Chèn es la imagen/representación [de] Yax Chit Witz'.

\section{Los wahy de Kaan}

Los wahy entendidos como entidades anímicas asociadas a las élites mayas (Moreno Zaragoza, 2011), son muy representados en materiales cerámicos de estilo códice en 


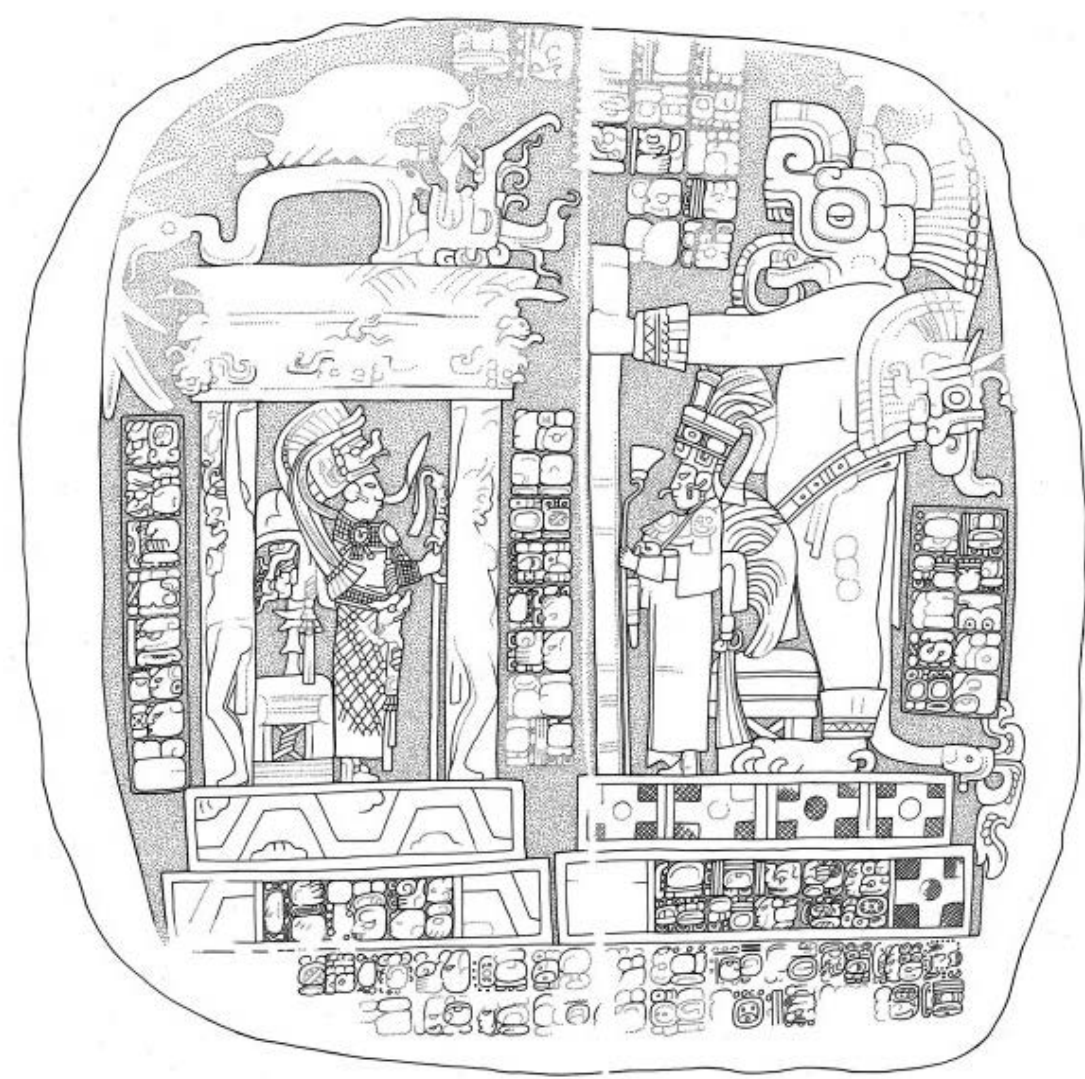

Figura 8. Panel 6 de La Corona (según Stuart en Taube, Saturno, Stuart y Hurst, 2013, Figura 1).
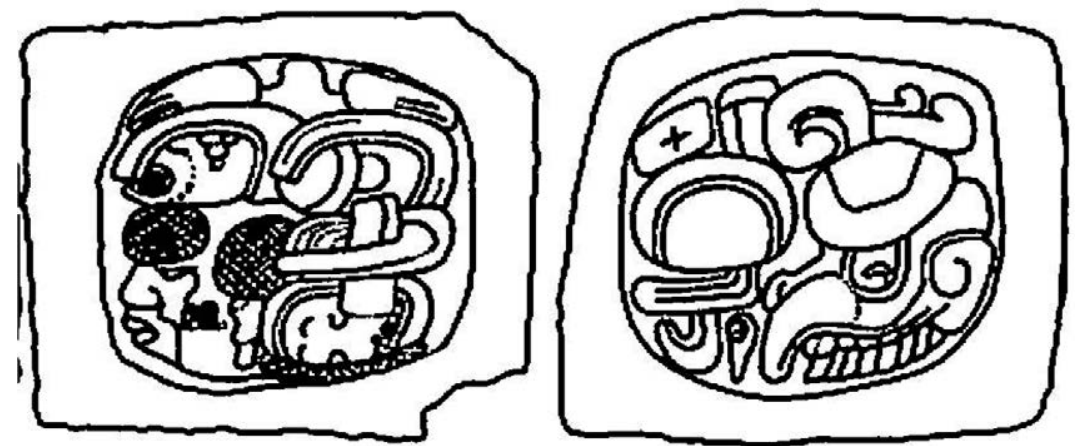

Figura 9. Piedras Cy D de Yo’okop (según Shaw et al., 2001, pp. 21-22).

cuya iconografía suelen adoptar formas de animales, entre otros seres, pareciendo ser habitantes del nivel sobrenatural utilizados por los gobernantes del Clásico para recorrer dicho plano existencial (Moreno Zaragoza, 2014, p. 394). Además de estas entidades, Erik Velásquez García ha propuesto la presencia de otras conocidas como o’hlis, corazón, centro o ánimo, "este controlaba la interioridad y el pensamiento, se identificaba con la vida, la energía, el espíritu, el centro y el ánimo; en él residía la individualidad y la personalidad (...) en él se ubicaban las fuerzas esenciales para la vida" (Velásquez García, 2009, p. 521) y b’aahis la cual se concentraba en la cabeza, "ésta constituía el centro de la individualidad y de los actos reflexivos; en ella se concentraba el coraje, el valor y la autoridad (...)” (Velásquez García, 2009, p. 567). 


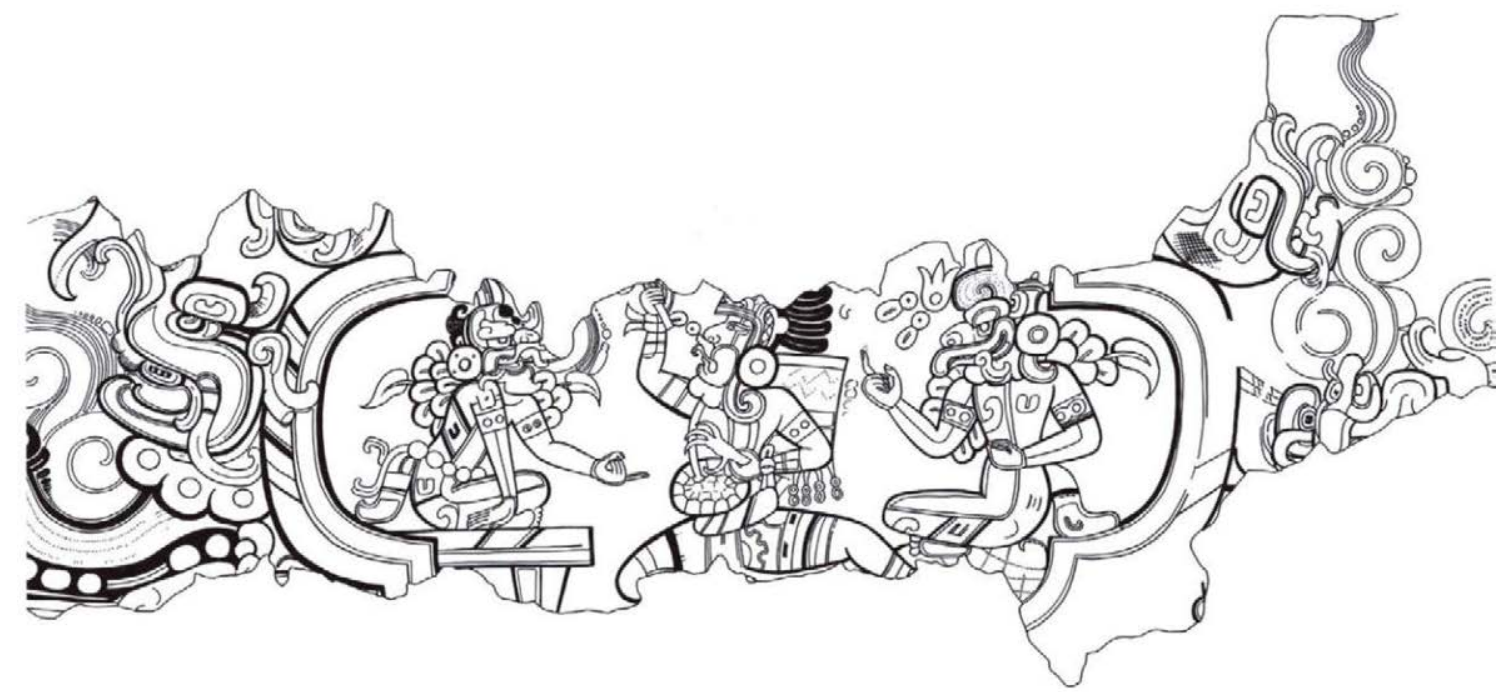

Figura 10. Escena del mural del Edificio Sub1-A de San Bartolo (según Hurst en Taube, Saturno, Stuart, y Hurst, 2010, p. 46).
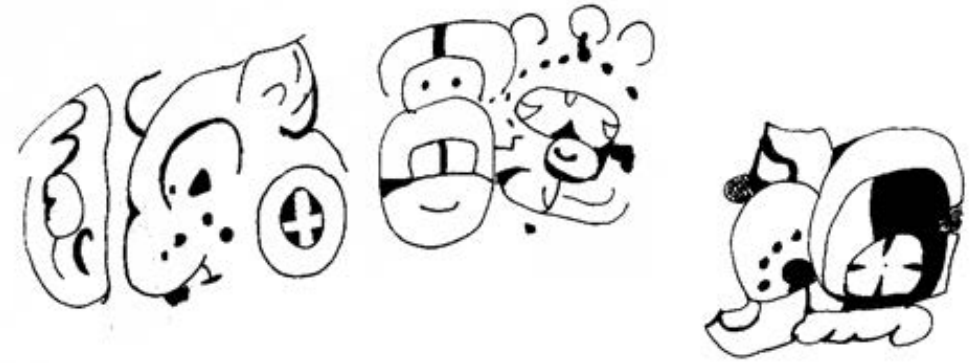

Figura 11. Registro escrito en la máscara de la Tumba 4 de la subestructura II-B de Calakmul (dibujo del autor).

Por su parte, los wahy podían ser "utilizados" por los gobernantes a los que estaban vinculados para atacar a sus enemigos, lo que llevó a Stuart a expresarse en términos de "brujería" refiriéndose al uso que se hacía de estos wahy y a "demonios" al describir a los entes mismos (Stuart, 2005), por ello, los ajawtaak eran poseedores del título de wahyaw, 'señor que llama a los wahyis' (Moreno Zaragoza, 2011, p. 137). Estos entes se han ligado a diversas dinastías del periodo Clásico al reconocerse entre los jeroglíficos que componen las narrativas, el glifo emblema asociado a los antropónimos de los wahy representados en las vasijas cerámicas. En el caso de la dinastía Kaan se pueden identificar varias entidades anímicas ligadas al glifo de la Cabeza de Serpiente destacando, para este trabajo, las que adquieren forma de serpiente.

\section{a) Xukub Chij Chan, "la serpiente-venado cornuda"}

Son dos las vasijas cerámicas estilo códice en las que se encuentran las referencias directas a estos wahy de los Kaan, catalogadas como K531 y K7794. En el primer ejemplo, el glifo emblema aparecerá en dos ocasiones nombrando a dos entes diferentes de los cuales uno de ellos volverá a ser aludido en el objeto K7794.

En la vasija K531 (Figura 12) se representa una escena que forma parte del grupo de las denominadas como "festines macabros" (Velásquez García, 2009, p. 630), en los 


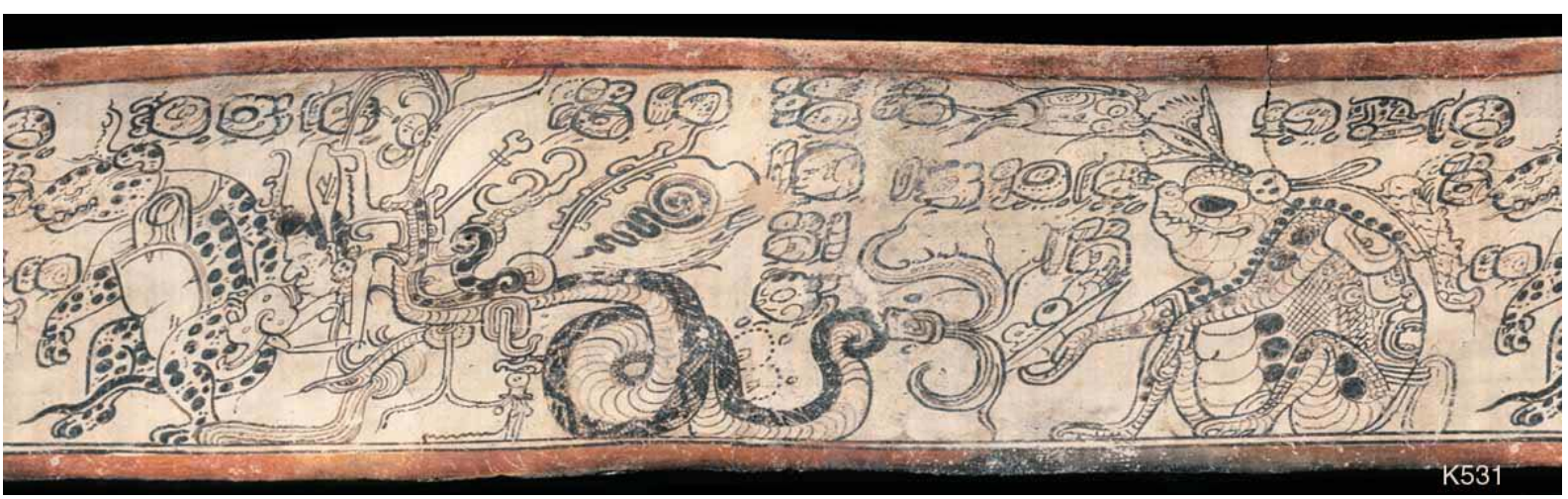

Figura 12. Vasija cerámica K531@ Justin Kerr.

cuales las entidades anímicas de los gobernantes del Clásico, en este caso de los Kaan, parecen haber devorado a los wahy de sus enemigos en el plano sobrenatural. La imagen se conforma de cuatro figuras, a la izquierda de la composición se encuentra un gran jaguar de pie sobre sus dos patas traseras a quien Grube y Nahm (Grube y Nahm, 1994) lo identificaron como un aspecto del dios jaguar del inframundo: lleva lo que podría ser un nenúfar en su cabeza y se distingue un nudo en su cuello que quizá fuese parte de la atadura de una capa, además se puede diferenciar en su estómago lo que parece el logograma $K^{\prime} I N$ que se liga con su nombre. Gracias a la secuencia jeroglífica que lo acompaña sabemos su antropónimo y su filiación dinástica: ?-[K'IN]-TAN-la BOLAY?TE'-la? u-WAY-ya? K'UH-ka-KAN-AJAW ba-ku-la, k'inta[h]nalbolay te' uwahyk'uh[ul] Kaanajawbakul. Cabe destacar la presencia del extraño compuesto jeroglífico de bakul (el cual no discutiremos en este escrito) que podría funcionar como un título asociado a los k'uhulajawtaak que detentan el control de los wahy representados.

En la parte central de la vasija se representa un gran ofidio barbado, con lo que parecen ser largas orejas, del cual se asoma entre sus fauces la deidad conocida como el dios de los venados, Wuk Sip (Grube, 2012, p. 139), soplando una concha que porta en sus manos. La secuencia narrativa lo identifica con el nombre de Xukub Chij Chan, "cuerno/asta venado serpiente", wahy del k'uhul Kaan ajaw en curso y bakul. En la parte derecha de la composición se puede distinguir un ser zoomorfo, un gran anfibio que carga un plato con lo que parecen ser restos de una mano y un globo ocular, entre otros elementos.

De nuevo en la vasija K7794 (Figura 13) se representa al mismo wahy de Kaan, Xukub Chij Chan, identificado tanto por su nombre jeroglífico como por su iconografía y por la presencia del glifo emblema de la Cabeza de Serpiente, del cual emergerá, como en el ejemplo anterior, el dios de los venados soplando su concha; también porta el título de bakul. En esta cerámica se acompaña de un wahy en forma aviar quizá relacionado con un ajaw de la entidad política de Chatahn, aunque sus jeroglíficos nominales son de difícil lectura. Cabe destacar que el ofidio Xukub Chij Chan, la serpiente venado cornuda, es un ser cuyo concepto hunde sus raíces en tiempos muy antiguos al ser una entidad representada también en otras regiones culturales como en Teotihuacán, lo que llevo a Helmke y Nielsen a tildarlo de "pan-mesoamericano" al encontrar también referencias posteriores entre los mexicas (Helmke y Nielsen, 2009, p. 78). Lo particular de este ser es que se relaciona con el ámbito oscuro, propagador de enfermedades e incluso muerte en crónicas coloniales (Helmke y Nielsen, 2009, p. 80). Por ello, este wahy se representa acompañado de otros poderosos entes sobrenaturales malignos como el gran sapo de la vasija K531, cuyo nombre compuesto por los signos $\boldsymbol{a}$-ma-la podría estar haciendo referencia al término am, que en maya yucateco significa 'escuerzo o sapo ponzoñoso' (Grube y Nahm, 1994), o el ser aviar del vaso K7794 cuyo cráneo parece estar descarnado y que podría estar asociado a un tipo de enfermedad y muerte. Además, debo señalar 


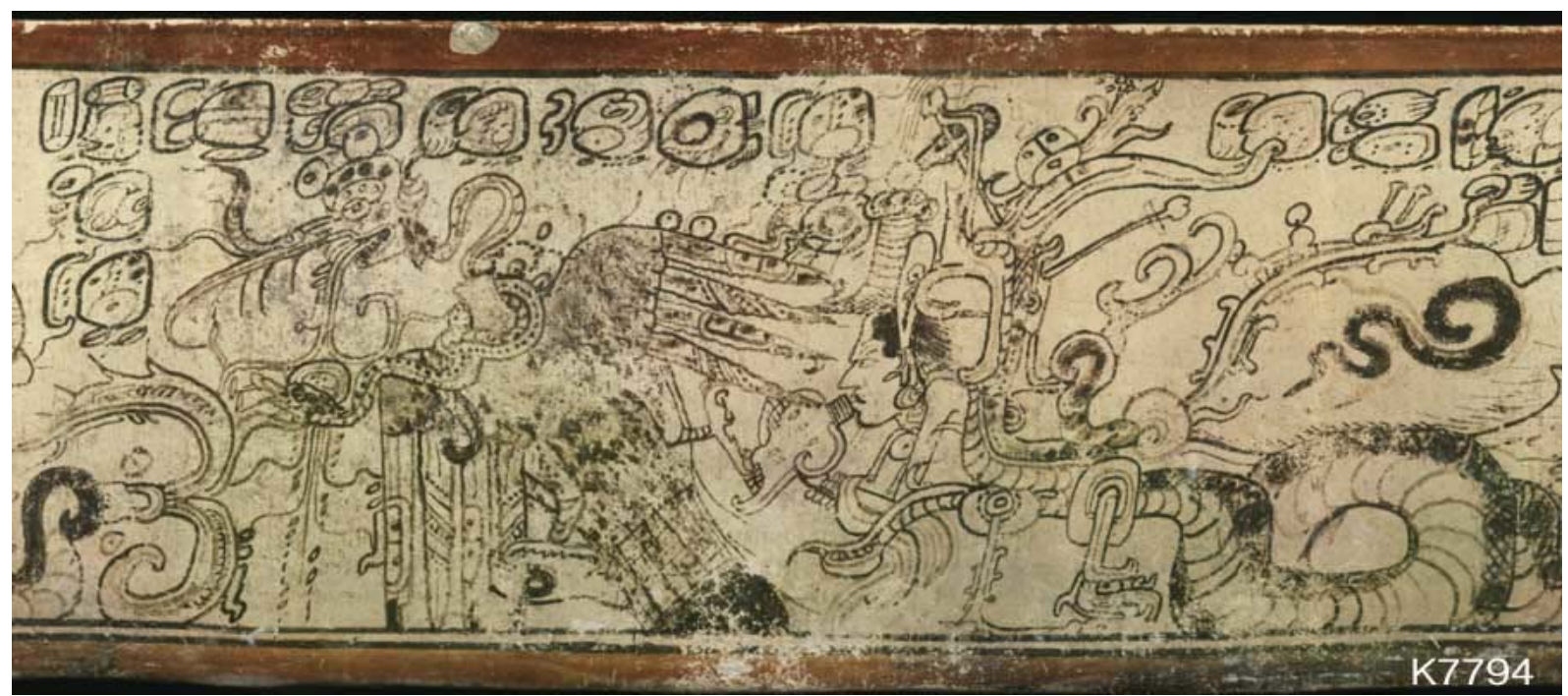

Figura 13. Vasija cerámica K7794 @ Justin Kerr.

que existen otros dos ejemplos cerámicos en los que se representa dicha entidad, son los vasos K2572 y K1384, pero en ellos no aparece asociada al glifo emblema de la Cabeza de Serpiente. Sin embargo, por el trazo del dibujo y los elementos iconográficos representados podrían formar parte del conjunto de materiales suntuarios vinculados a las entidades corporativas que conformaban la corte Kaan.

\section{Consideraciones finales}

Desde los primeros monumentos esculpidos asociados al grupo de poder, la dinastía Kaan tomó como signo de identidad a uno de los animales con mayor carga simbólica en el imaginario colectivo del Clásico: la serpiente. Además, los gobernantes tempranos revistieron de autoridad a su glifo emblema al modificar su lectura con el complemento $\boldsymbol{k a}$-, legitimándose de este modo como un linaje ligado a raíces muy antiguas.

Como se mostró, los ajawtaak se asociaron a tres ofidios sobrenaturales que envolvían de poder a la dinastía tanto en el plano terrenal como sobrehumano: Waxaklajunubaah Chan, la Serpiente acuática y Xukub Chij Chan. Como wahyaw, los gobernantes de Kaan controlaban a entidades anímicas que tomaban diversas apariencias sobrenaturales con las que quizá podrían derrotar a sus contrarios en un plano supraterrenal. Por ello, las vasijas tipo códice decoradas con dicha temática eran, en mi opinión, una manera de reivindicar la vinculación a estos poderosos entes durante el complicado contexto histórico del Clásico Tardío, revistiéndose los ajawtaak de la Cabeza de Serpiente de un mayor poder. No en vano, el aumento de la utilización de símbolos de poder ligados a una tradición anterior, junto con el incremento significativo de representaciones iconográficas de wahy, mitos y la presencia de tres deidades importantes del panteón maya, la Serpiente acuática, Chaahk y el dios del Maíz, durante el Clásico Tardío, parecen indicar un programa de revalorización dinástica como respuesta a una crisis político-social que alcanzó sus cotas más críticas con Yuhkno’m Took' Káwiil. De esta manera, los gobernantes tardíos eran la representación terrenal de las poderosas entidades sobrenaturales $\mathrm{y}$, además, controlaban a los temidos wahy propagadores de enfermedades y muerte como Xukub Chij Chan.

En este sentido, cabe destacar que la fecha en la que Yuhkno'm Yihchaak K'ahk' está tomando los atributos de la serpiente de la guerra teotihuacana Waxaklajunubaah 
4. Sigo las hipótesis de la doctora Ana García Barrios (2008) pero, a la vez, soy consciente de las interpretaciones de los investigadores Ramón Carrasco Vargas y María Cordeiro quienes consideran que el personaje representado no sería la deidad Chaahk, sino más bien: "el ch'ulel, entidad anímica de los mayas que reside en el Xibalbá lo mismo que en los seres humanos, plantas y animales" (Carrasco Vargas y Cordeiro, 2014, p. 43).
Chan en La Corona, se corresponde con el año 692 d.C. Un año antes, en la cerámica K5453, se representa a un emisario del gobernante calakmuleño haciendo entrega de una serie de dádivas al ajaw de Tikal Jasaw Chan K'awiil, su enemigo acérrimo, en su corte de Topoxté y pocos años después tal y como se indica en el Dintel 3 del Templo I de Tikal, el ejército del gobernante de Calakmul cayó derrotado a manos de las fuerzas tikaleñas sufriendo una gran humillación al perder en el enfrentamiento a una de sus principales deidades tutelares. Por ello, a pesar de que los ajawtaak de Kaan utilizaron un meditado programa iconográfico en su favor, sobre todo desde la segunda mitad del siglo VII d.C., basado en el ensalzamiento militar y en el dominio de poderosas entidades sobrenaturales, la realidad político-social de las Tierras Bajas mayas estaba experimentando un proceso de cambio, que para los gobernantes del glifo emblema de la Cabeza de Serpiente será totalmente adverso y al que intentaron hacerle frente.

En cuanto al simbolismo de la serpiente, no debemos olvidar que hoy en día, en la región de las Tierras Altas de Guatemala, tal y como señaló García Barrios (2016, p. 18): "todavía los mayas tzutujiles piensan que los jaguares y las serpientes son los guardianes de las cuevas, lugares sagrados donde moran los ancestros y deidades". Dichos conceptos, reminiscencias de un tiempo anterior, quedaron plasmados en Calakmul en el interior de la Estructura II donde se halló la Subestructura II-c fechada para el periodo Preclásico, cuya entrada enmarcada por un gran friso estucado y policromado está flanqueada por dos grandes mascarones con rasgos de jaguar, otro de los animales vinculado a los gobernantes de Kaan en su representación como wahy. Además, la figura central del friso parece representar a $C h a a h k^{4} y$ a sus lados se disponen seres serpentinos, mientras que unos elementos iconográficos superiores en forma de gancho simbolizan la entrada a la cavidad. Una cueva artificial construida a partir de una bóveda de arco de cañón corrido que fue cubierta en posteriores modificaciones estructurales.

La presencia del término chèn, 'cueva', en la vasija tipo códice 23 de Calakmul como indicó García Barrios (2011, p. 86), junto con el glifo emblema de Kaan, podría referirse a la expresión ochcheen, 'entrar a la cueva de Kaan' quizá aludiendo a esta construcción que, aunque "escondida", pudo ser conocida por los gobernantes del glifo emblema de la Cabeza de Serpiente tras instalarse en Calakmul y tal vez sirvió para reforzar el mito del origen dinástico durante el Clásico Tardío, en el que serpientes (y también jaguares) forman parte del registro iconográfico y jeroglífico de tan importante dinastía. 


\section{Q Referencias citadas}

" Baron, J. (2013). Patrons of La Corona: Deities and Power in a Classic Maya Community. (Tesis Doctoral inédita), Universidad de Pennsylvania, Estados Unidos.

"Barthel, T. (1968). El complejo “emblema”. En Estudios de Cultura Maya, Volumen VII (pp. 159-193). Ciudad de México.

» Beliaev, D. (2015). Taller de Epigrafía especializada. Trabajo presentado en II Encuentro de Gramatología. Ciudad de México, México.

» Berlin, H. (1958). El glifo «emblema» de las inscripciones mayas. Journal de la Société des Américanistes, 47, 111-119.

" Bernal Romero, G. (2015). El logograma T514, YEJ, “filo". Un comentario epigráfico. Manuscrito inédito.

" Biró, P. (2011). The Classic Maya Western Region: A History. Oxford: Archaeopress.

"Carrasco Vargas, R. (2012). Cronología e historia. En R. Martínez Vera (Coord.), Calakmul. Patrimonio de la humanidad (pp. 75-84). Ciudad de México: Grupo Azabache.

»Carrasco Vargas, R. y Cordeiro, M. (2014). El origen de la montaña. Arqueología Mexicana, 128(XXII), 41-45.

"Clancy, F. (1994). The Classic Maya Ceremonial Bar. Anales del Instituto de Investigaciones Estéticas, 65(XVI), 7-45.

»D'arcy Harrison, P. (2008). Animales como nombres de familias reales en Tikal y algunas consideraciones sobre Calakmul. Mayab, 20, 109-124.

"De la Garza, M. (2001). La serpiente en la religión maya. En Y. González Torres (Coord.), Animales y plantas en la cosmovisión mesoamericana (pp. 145-157). Ciudad de México: Plaza y Valdés.

»Domínguez, M. R. y Folan, W. (1996). Calakmul: México. Aguadas, bajos, precipitaciones y asentamiento en el Petén campechano. En J. P. Laporte y H. Escobedo (Eds.), IX Simposio de Investigaciones Arqueológicas en Guatemala (pp. 147-173). Guatemala: Museo Nacional de Arqueología y Etnología.

"Esparza Olguín, O. Q. (2012). Las escaleras jeroglíficas de El Resbalón, Quintana Roo. Estudio de un conjunto de inscripciones mayas del Clásico Temprano. (Tesis de Maestría inédita), Universidad Nacional Autónoma de México, México.

"Esparza Olguín, O. Q. y Pérez Gutiérrez, V. (2009). Archaeological and Epigraphic Studies in Pol Box, Quintana Roo. The PARI Journal, 3(IX), 1-16.

» Estrada-Belli, F., Tokovinine, A., Foley, J., Hurst, H., Ware, G., Stuart, D. y Grube, N. (2009). A Maya Palace at Holmul, Peten, Guatemala and the Teotihuacan "Entrada": Evidence from Murals 7 and 9. Latin American Antiquity, 20(1), 228-259.

» Freidel, David A., Schele, L. y Parker, J. (1993). Maya Cosmos: Three Thousand Years on the Shaman's Path. Nueva York: W. Morrow.

" García Barrios, A. (2008). Chaahk, el dios de la lluvia en el periodo Clásico Maya: aspectos religiosos y políticos. (Tesis Doctoral inédita), Universidad Complutense de Madrid, España.

" García Barrios, A. (2016). Cuevas y montañas sagradas: espacios de legitimación y ritual del dios maya de la lluvia. En R. Romero Sandoval (Ed.), Cuevas y cenotes mayas. Una mirada multidisciplinaria (pp.15-56). Ciudad de México: Universidad Nacional Autónoma de México. 
» Grube, N. (2012). A Logogram for SIP, “Lord of the Deer”. Mexicon, XXXIV, 138-141.

"Grube, N. y Nahm, W. (1994). Census of Xibalbá. A Complete Inventory of Way Characters on Maya Ceramics. En J. Kerr (Ed.), The Maya Vase Book 4 (pp. 686-715). New York: Kerr Associates.

» Helmke, C. (2012). Mythological Emblem Glyphs of Ancient Maya Kings. En C. Helmke yJ. Zralka (Eds.), Contributions in New World Archaeology 3 (pp. 91-126). Cracovia: Jagiellonian University.

" Helmke, C. y Kupprat, F. (2017). Los glifos emblema y los lugares sobrenaturales: el caso de Kanu'l y sus implicaciones. Estudios de Cultura Maya, L, 95-135.

» Helmke, C. y Awe, J. (2016). Sharper than a Serpent's Tooth: A Tale of the Snake-head Dynasty as Recounted on Xunantunich Panel 4. The PARI Journal, 2(XVI), 1-22.

» Helmke, C. y Nielsen, J. (2009). Hidden Identity \& Power in Ancient Mesoamerica: Supernatural Alter Egos as Personified Diseases. Acta Americana, 17, 49-98.

" Kaufman, T. y Justeson, J. (2003). A Preliminary Mayan Etymological Dictionary. http:// www.famsi.org/reports/01051/pmed.pdf (Acceso: 18 de abril, 2018).

» Kettunen, H. y Davis II, B. V. (2004). Snakes, Centipedes, Snakepedes and Centiserpents: Conflation of Liminal Species in Maya Iconography and Ethnozoology. Wayeb Notes, 9, 1-42. http://www.wayeb.org/notes/wayeb_notesooog.pdf (Acceso: 18 de abril, 2018).

» Kettunen, H. y Helmke, C. (2013).Water in Maya Imagery and Writing. En C. Helmke y J. Zralka (Eds.), Contributions in New World Archaeology 5 (pp. 17-38). Cracovia: Jagiellonian University.

» Kettunen, H. y Helmke, C. (2014). Introduction to Maya Hieroglyphs. Bratislava: Wayeb, Sociedad Europea de Mayistas. http://wayeb.org/download/resources/wh2014english. pdf (Acceso: 18 de abril, 2018).

» Kerr, J. y Kerr, B. http://www.mayavase.com/index.html (Acceso: 23 de abril, 2019)

"Lacadena, A. y Wichmann, S. (2002). The Distribution of Lowland Maya Languages in the Classic Period. En V. Tiesler, R. Cobos y M. Greene (Eds.), La organización entre los mayas. Memoria de la tercera mesa redonda de Palenque (pp. 275-314). Ciudad de México: Instituto Nacional de Antropología e Historia, Universidad Autónoma de Yucatán.

" Law, D. y Stuart, D. (2017).Classic Mayan. An overview of language in ancient hieroglyphic script. En J. Aissen, N. England y R. Zavala Maldonado (Eds.), The Mayan Languages (pp. 128-172). Londres: Routledge.

» Marcus, J. (1973). Territorial Organization of the Lowland Classic Maya. Science, New Series, 180, 911-916.

» Martin, S. (1997). The Painted King List: A Commentary on Codex-Style Dynastic Vases. En J. Kerr y B. Kerr (Eds.), The Maya Vase Book Vol. 5 (pp. 847-883). Nueva York: Kerr Assocites.

"Martin, S. (2008). Wives and Daughters on the Dallas Altar http://www.mesoweb.com/ articles/martin/Wives\&Daughters.pdf (Acceso: 18 de abril, 2018).

" Moreno Zaragoza, D. (2011). Los espíritus del sueño. Wahyis y enfermedad entre los mayas del periodo Clásico. (Tesis de Licenciatura inédita), Escuela Nacional de Antropología e Historia, México.

» Moreno Zaragoza, D. (2014). La transformación onírica a través de las representaciones de wahyis del Clásico. En B. Arroyo, L. Méndez Salinas y A. Rojas (Eds.), XXVII Simposio de Investigaciones arqueológicas en Guatemala (pp. 391-401). Guatemala: Ministerio de Cultura y Deportes, Instituto de Antropología e Historia, Asociación Tikal. 
» Nalda, E. (2004). Dzibanché. El contexto de los cautivos. En E. Nalda (Ed.), Los cautivos de Dzibanché (pp. 12-58). Ciudad de México: Instituto Nacional de Antropología e Historia.

» Prager, C. (2004). A Classic Maya Ceramic Vessel from the Calakmul Region in the Museum zu Allerheiligen, Schaffhausen, Switzerland. Human Mosaic, 35(1), 31-40.

»Reents-Budet, D., Boucher, S., Palomo, Y., Bishop, R. y Blackman, J. (2011). Cerámica de estilo códice: nuevos datos de producción y patrones de distribución. En B. Arroyo, L. Paiz, A. Linares y A. Arroyave (Eds.), XXIV Simposio de Investigaciones arqueológicas en Guatemala (pp. 832-846). Guatemala: Museo Nacional de Arqueología y Etnología.

»Shaw, J., Johnstone, D. y Krochok, R. (2001). Final Report of the 2000 Yo'okop Field Season: Initial Mapping and Surface Collections. FAMSI: Fundación para el Avance de los Estudios Mesoamericanos Inc. http://www.famsi.org/reports/99016 (Acceso: 18 de abril, 2018).

»Stuart, D. (2000). The Arrival of the Strangers: Teotihuacan and Tollan in Classic Maya History. En D. Carrasco, L. Jones y S. Sessions (Eds.), Mesoamerica's Classic Heritage: from Teotihuacan to the Aztecs (pp.465-513). Niwot: Colorado University Press.

»Stuart, D. (2005). The Way Beings. En Glyphs on Pots Decoding Classic Maya Ceramics. A section of the 2005 edition of the Sourcebook for the 29th Maya Meetings at Texas. Austin: University of Texas. https://decipherment.files.wordpress.com/2013/og/ stuartceramictexts.pdf (Acceso: 18 de abril, 2018).

»Stuart, D. (2007). Reading the Water Serpent as WITZ', https://decipherment.wordpress. com/2007/o4/13/reading-the-water-serpent/ (Acceso: 23 de abril, 2019).

»Stuart, D. (2012). Notes on a New Text from La Corona, https://decipherment.wordpress. com/2012/06/30/notes-on-a-new-text-from-la-corona/ (Acceso: 30 de junio de 2012).

»Stuart, D. (2013). New Drawing of a La Corona Panel, https://decipherment.wordpress. com/2013/01/23/new-drawing-of-a-la-corona-panel/ (Acceso: 23 de abril de 2019)

» Taube, K. (1992). The Temple of Quetzalcoatl and the Cult of Sacred War at Teotihuacan. Res: Anthropology and Aesthetics, 21, 53-87.

» Taube, K., Saturno, W., Stuart, D. y Hurst, H. (2010). Los murales de San Bartolo, El Petén, Guatemala, parte 2: E mural poniente. Ancient America, 10, 1-110.

» Thompson, J. E. (1962). A Catalog of Maya Hieroglyphs. Norman: University of Oklahoma Press.

»Velásquez García, E. (2007). La máscara de “rayos X”. Historia de un artilugio iconográfico en el arte maya. Anales del Instituto de Investigaciones Estéticas, 90(XXIX), 7-36.

»Velásquez García, E. (2009). Los vasos de la entidad política de 'lk’: Una aproximación histórico-astística. Estudio sobre las entidades anímicas y el lenguaje gestual y corporal en el arte maya clásico. (Tesis Doctoral inédita), Universidad Nacional Autónoma de México, México.

»Wren, L. y Nygard, T. (2005). Witnessed at Yo'okop: Images and Texts of Rulers in a Watery Realm. En J. Shaw y J. Mathews (Eds.), Quintana Roo Archaeology (pp. 166-18o). Tucson: Universidad de Arizona.

»Zender, M. y Stone, A. (2011). Reading Maya Art. A Hieroglyphic Guide to Ancient Maya Painting and Sculpture. Londres: Thames and Hudson. 